\title{
Possible Involvement of Anorexigenic Protein, Nesfatin/Nucleobindin-2 (NUCB2) in Blood Pressure Regulation by Regulating Water Reabsorption in Renal Collecting Duct
}

\author{
Hiroyuki Shimizu $^{1^{*}}$ and Aya Osaki ${ }^{2}$ \\ ${ }^{1}$ Department of Diabetes and Endocrinology, International University of Health and Welfare (IUHW) Hospital, Nasushiobara, Japan \\ ${ }^{2}$ Department of Medicine and Molecular Science, Gunma University Graduate School of Medicine, Maebashi, Japan \\ ${ }^{*}$ Corresponding author: Hiroyuki Shimizu, Department of Diabetes and Endocrinology, International University of Health and Welfare Hospital, Iguchi 537-3 \\ Nasushiobara 329-2763 Japan, Tel: 81-287-37-2221, Extn. 2731, Fax: 81-287-39-3001; E-mail: hshimizu@iuhw.ac.jp
}

Rec date: Oct 04, 2014, Acc date: Nov 03, 2014, Pub date: Nov 05, 2014

Copyright: (C) 2014 Shimizu H, et al. This is an open-access article distributed under the terms of the Creative Commons Attribution License, which permits unrestricted use, distribution, and reproduction in any medium, provided the original author and source are credited.

\begin{abstract}
Nesfatin/nucleobindin-2 (NUCB2), a precursor protein of anorexigenic protein, nesfatin-1, is ubiquitously expressed in the body. The finding that peripheral administration of nesfatin-1 increases blood pressure indicates a possible involvement of nesfatin-1 in the regulation of blood pressure. The present studies were undertaken to investigate a possible involvement of nesfatin/NUCB2 in the regulation of blood pressure. The immunoreactivity against nesfatin/NUCB2 was selectively found in vascular endothelial cells of aorta, pulmonary artery and renal artery, cardiac muscle and skeletal striated muscle cells, but not in vascular smooth muscle cells at all. Furthermore, the immunoreactivity against nesfatin/NUCB2 was selectively found in renal collecting duct cells, which contain aquaporin (AQP)-2 and/or epithelial sodium channel (ENaC). In medullary collecting ducts, cells expressing nesfatin/ NUCB2 co-expressed AQP-2, but did not co-expressed AQP-2 in renal cortical collecting ducts. On the other hand, collecting ductal cells expressing ENaC were totally compatible with those expressing nesfatin/NUCB2 in both renal medullary and cortical collecting ducts. Thus, there is a possibility that nesfatin/NUCB2 is involved in the regulation of blood pressure through increased water reabsorption in the kidney.
\end{abstract}

Keywords: Nesfatin; Nucleobindin-2; Epithelial sodium channel; Endothelial cell; Renal collecting duct

\section{Introduction}

Nesfatin/nucleobindin-2 (nesfatin/NUCB2), a precursor protein of anorexigenic protein, nesfatin-1, was found in the paraventricular nucleus of the hypothalamus [1]. Nesfatin/NUCB is ubiquitously expressed in the body, and the expression of nesfatin/NUCB2 in peripheral tissue is partially regulated by the hypothalamus via sympathetic nervous system [2]. Peripheral administration of nesfatin-1 suppressed food intake in mice, indicating that peripherally produced nesfatin-1 may regulate central nervous system [3]. Since circulating concentration of nesfatin/NUCB is correlated with body mass index, nesfatin/NUCB2 is supposed to involve in the development of metabolic syndrome [4].

Central administration of nesfatin-1 increases blood pressure, dependent upon $\alpha$-adrenergic system [5]. In contrast, we have recently demonstrated an involvement of peripherally produced nesfatin/ NUCB2 in the regulation of blood pressure in vivo, since peripheral administration of nesfatin- 1 increases blood pressure mediated by $\beta$ adrenergic mechanism in mice [6]. Nesfatin-1 could be a candidate for the treatment of obesity, if blood pressure elevation can be controlled [7]. The present studies were undertaken to investigate a possible involvement of nesfatin/NUCB2 in the peripheral regulatory system of blood pressure by evaluating local expression of nesfatin/NUCB2 in peripheral tissues related to the regulation of blood pressure.

\section{Materials and Methods}

\section{Animals}

Male Wistar rats weighing about $200 \mathrm{~g}$ and a male mouse at age of 10 week were obtained from Charles Rivers Japan, Inc., Tokyo, Japan. The animals were maintained on a 12 hour light/dark cycle and were allowed free access to food and water. Under inhalation anesthesia by isofane, a rat was sacrificed, and gastrocnemius muscle, heart, and kidney were dissected out, and immediately fixed in $10 \%$ formalin solution. On the dissection of the vessel, a rat was sacrificed, transcardially perfused by $0.9 \%$ sodium chloride solution, followed by the infusion of $10 \%$ formalin solution. The aorta, pulmonary and renal arteries were dissected out, and further fixed in $10 \%$ formalin solution. The experiment was performed at Kiryu University from April, 2010 to March, 2012, and the protocol of the experiment was approved by the local committee on animal welfare.

\section{Immunohistochemistry}

After all samples were embedded in paraffin, paraffin-embedded sections were dewaxed, and rehydrated. After boiling for $20 \mathrm{~min}$ in 10 $\mathrm{mM}$ citrate buffer ( $\mathrm{pH}$ 6.0), sections were cooled for 20 minutes at room temperature. Endogenous peroxidase activity was blocked through incubation with $3 \% \mathrm{H} 2 \mathrm{O} 2$ for 5 minutes, and sections were washed three times by $0.01 \mathrm{M}$ PBS ( $\mathrm{pH}$ 7.4). After incubated with primary antibody overnight at $4^{\circ} \mathrm{C}$, then washed in PBS, slides were incubated with secondary antibody (Histofine Simplestain Rat MAXPO, Nichirei Biosciences Inc., Japan) for 30 minutes at room temperature. Goat polyclonal antibodies against nesfatin/NUCB2 were 
Citation: Shimizu H, Osaki A (2014) Possible Involvement of Anorexigenic Protein, Nesfatin/Nucleobindin-2 (NUCB2) in Blood Pressure Regulation by Regulating Water Reabsorption in Renal Collecting Duct. J Cytol Histol 5: 291. doi:10.4172/2157-7099.1000291

Page 2 of 3

obtained from Santa Cruz Biotechnology (CA, U.S.A.), and rabbit polyclonal antibody against aquaporin (AQP)-2 and epithelial sodium channel (ENaC) from Abcam (U.K.).

\section{Real time-polymerase reaction (PCR)}

Vascular endothelial cells were isolated from the aorta of mouse, according to the method described previously [8]. A male ICR mouse was anesthetized with an intraperitoneal injection of $4 \mathrm{mg}$ of pentobarbital sodium. After anesthesia, abdomen and thorax are opened. The abdominal aorta is cut at the middle to release blood, and then perfused with $1 \mathrm{ml}$ of PBS containing $1000 \mathrm{U} / \mathrm{ml}$ of heparin from heart. The aorta is dissected from aortic arch to the abdominal aorta, and immersed in 20\% FBS-DMEM containing $100 \mathrm{U} / \mathrm{ml}$ of heparin. A $24 \mathrm{G}$ cannula is inserted into the proximal potion of aorta. After ligation at the site with a thread, the inside the lumen is briefly washed with serum-free DMEM. The other side is bound and filled with the solution containing collagenase type 2 (Worthington biochemical corporation, Lakewood, NJ, USA). After incubation for $45 \mathrm{~min}$ at $37^{\circ} \mathrm{C}$, endothelial cells are removed from the aorta by flushing $5 \mathrm{ml}$ of DMEM containing 20\% FBS. Total RNA was isolated from isolated vascular endothelial cells with Isogene (Nippon Gene, Tokyo, Japan), according to the manufacturer's protocol. Reverse transcription was performed using a PrimeScript $^{\oplus}$ RT reagent Kit (Takara Bio, Inc., Shiga, Japan). For quantitative PCR, cDNA fragments were amplified by TaqMan ${ }^{\circ}$ Gene Expression Master Mix (Applied Biosystems), according to the manufacturer's protocol. GAPDH was used as the internal control. Real-time PCR was performed with a Real-Time PCR System (Applied Biosystems). The cycling conditions were $95^{\circ} \mathrm{C}$ for 15 seconds and $60^{\circ} \mathrm{C}$ for 1 minute, for a total of 40 cycles. The amount of transcribed cDNA was normalized to GAPDH expression.

\section{Results}

As shown in Figure 1a, 1b, and 1c, the immunoreactivity against nesfatin/NUCB2 was found cardiac muscle and skeletal striated muscle cells, but not in vascular smooth muscle cells at all In addition, the immunoreactivity against nesfatin/NUCB2 was selectively found in vascular endothelial cells of aorta, pulmonary artery and renal artery (Figure 1d). The existence of nesfatin/NUCB2 in vascular endothelial cells was confirmed by PCR analysis in isolated endothelial cells obtained from the aorta of male ICR mouse.

Furthermore, the immunoreactivity against nesfatin/NUCB2 was selectively found in renal collecting duct cells, which contain AQP-2 and/or ENaC (Figure 2a). Cells expressing nesfatin/NUCB2 coexpressed with AQP-2 in all medullary collecting ducts, but with AQP-2 in only a small number of renal cortical collecting ducts (Figure $2 \mathrm{~b}$ ). In contrast, almost the collecting ductal cells expressing $\mathrm{ENaC}$ co-localized with those expressing nesfatin/NUCB2 in both renal medullary and cortical collecting ducts (Figure 2c). No obvious immunoreactivity against nesfatin/NUCB2 was found at all in the glomerulus, proximal tubular cells, and renal interstitial cells.

\section{Discussion}

The present study demonstrated the selective expression of nesfatin/NUCB2 in peripheral tissues related to the regulation of blood pressure. First, nesfatin/NUCB2 was expressed in vascular endothelial cells. Our recent data indicate that nesfatin-1 regulates blood pressure by directly modifying vascular contractility via a $\beta$-adrenergic system [6]. It has been recently shown that nesfatin-1 has the incremental contribution to hypertension risk prediction, and plasma nesfatin-1 level in hypertension patients with microalbuminuria are significantly higher than those without microalbuminuria patients [9]. Although a direct action of nesfatin-1 or nesfatin/NUCB2 has not be shown in vascular endothelial cells, nesfatin/NUCB2 or nesfatin-1 secreted from vascular endothelial cells may be involved in the regulation of blood pressure by directly modifying the contractility of vascular smooth muscles as a paracrine factor.

Next, nesfatin/NUCB2 was selectively expressed in renal collecting ductal cells which co-express ENaC and/or AQP-2. Nesfatin/NUCB2 co-localized with $\mathrm{ENaC}$ in both cortical and medullary collecting duct, but with AQP-2 only in medullary collecting duct.

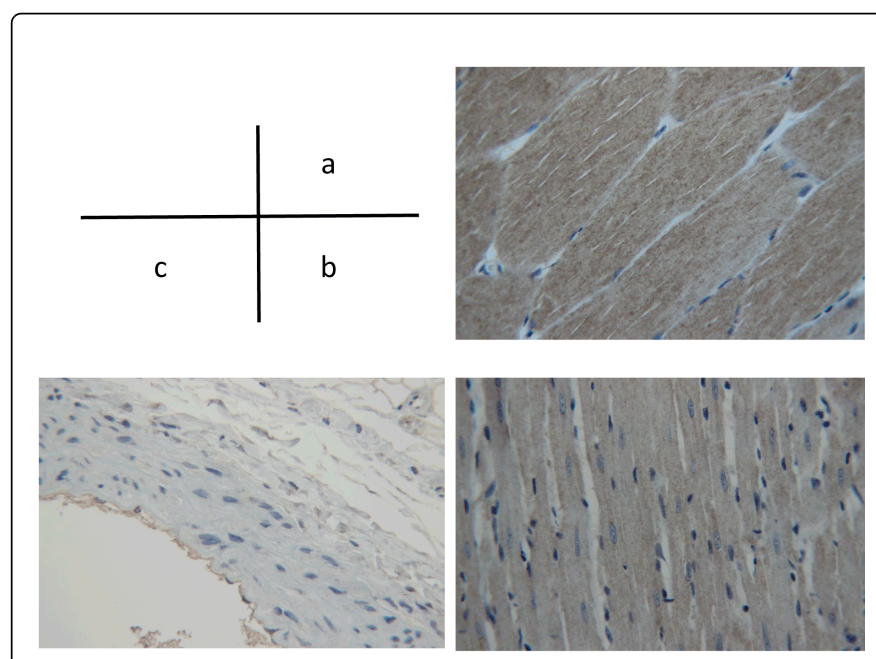

Figure 1(a-c): Immunoreactivity against nesfatin/NUCB2 in striated muscle (a), cardiac muscle (b), vascular smooth muscle (c).

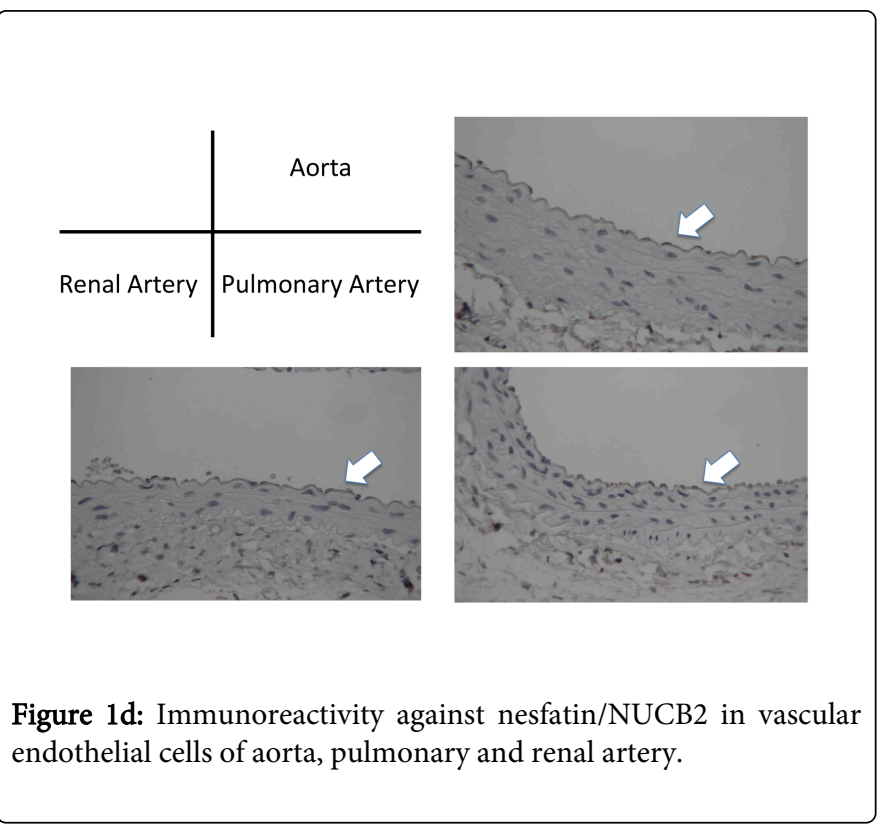


Citation: Shimizu H, Osaki A (2014) Possible Involvement of Anorexigenic Protein, Nesfatin/Nucleobindin-2 (NUCB2) in Blood Pressure Regulation by Regulating Water Reabsorption in Renal Collecting Duct. J Cytol Histol 5: 291. doi:10.4172/2157-7099.1000291

Page 3 of 3

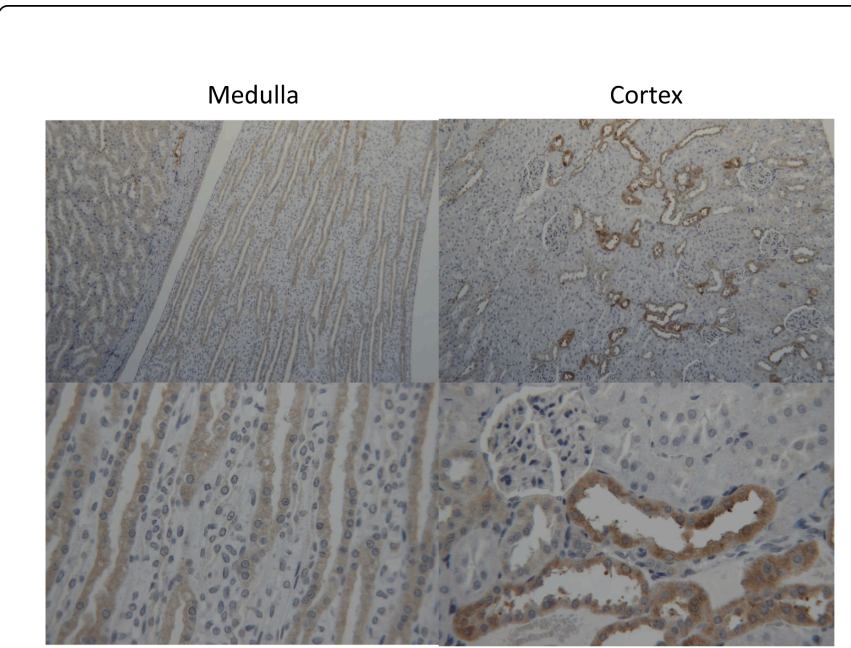

Figure 2a: Immunoreactivity against nesfatin/NUCB2 in renal medulla and cortex.

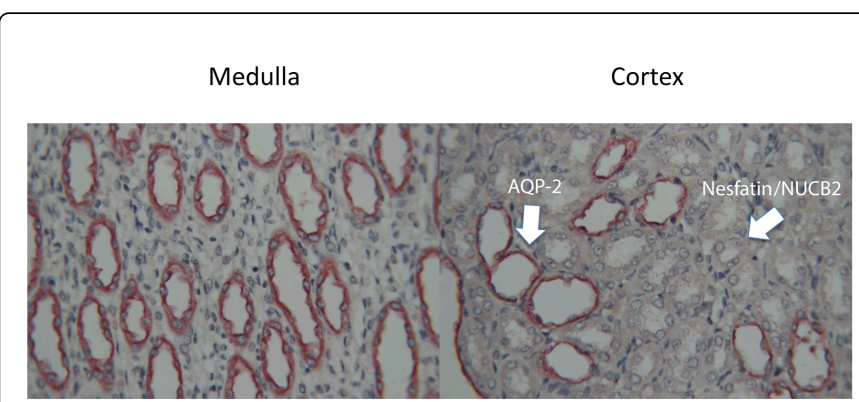

(double immunostaining against Nesfatin/NUCB2 (brown) x AQP-2 (red))

Figure 2b: double immunostaining again nesfatin/NUCB2 (stained in brown) and auqporin-2 (AQP-2; stained in red).

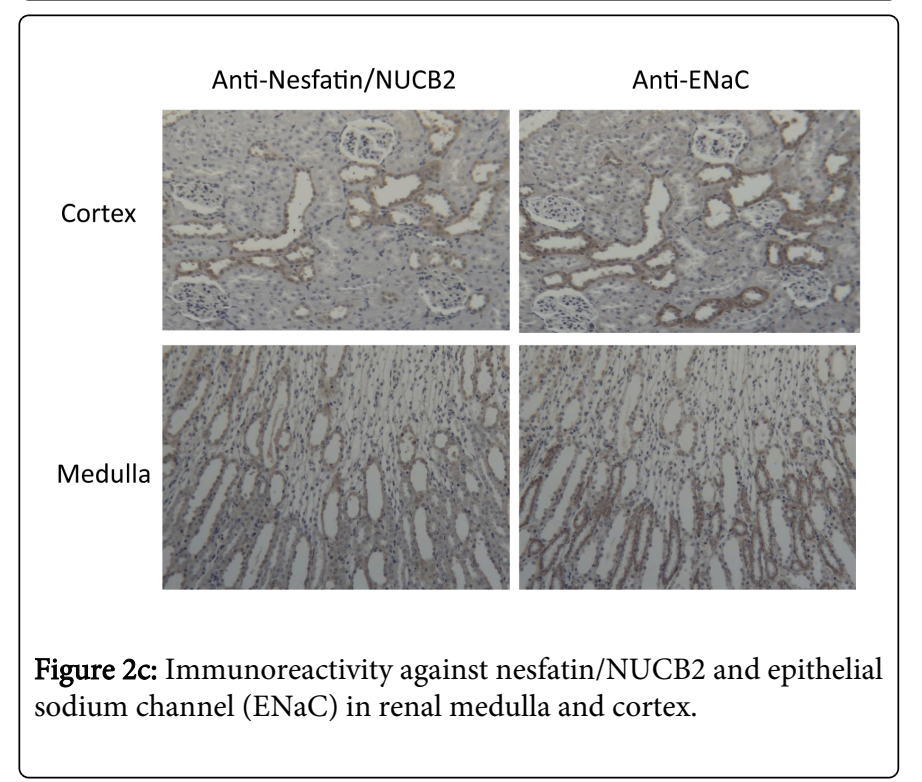

$\mathrm{ENaC}$ is involved in the regulation of sodium and water reuptake in renal epithelial cells that line the distal part of the renal tubule [10], and contribute to the regulation of blood pressure by modifying water retention in the body [11]. Circulating nesfatin/NUCB2 concentration has been reported to be significantly correlated with body mass index [4]. Obese people with metabolic syndrome are associated with saltsensitive hypertension, and hyperaldosteronism may be caused at least partially by certain adipocyte-derived factors [12]. It is supposed that an increase of circulating nesfatin/NUCB2 in the patients with obesity may reflect the increased production of nesfatin/NUCB in the kidney, and that increased nesfatin/NUCB2 may be partially involved in the elevation of blood pressure in obese people.

Thus, the present study raised a possibility that nesfatin/NUCB2 is involved in the development of hypertension in obese patients through vasoconstriction and increase of plasma volume by increased water reabsorption in the kidney.

\section{Source of Funding}

This work was supported in part by the Japanese Ministry of Education, Culture, Sports, Science and Technology, Grant-in-Aid for Scientific Research on Innovative Areas (Research in a proposed research area) "Molecular Basis and Disorders of Control of Appetite and Fat accumulation" for HS.

\section{References}

1. Oh-I S, Shimizu H, Satoh T, Okada S, Adachi S, et al. (2006) Identification of nesfatin-1 as a satiety molecule in the hypothalamus. Nature 443: 709-712.

2. Osaki A, Shimizu H, Ishizuka N, Suzuki Y, Mori M, et al. (2012) Enhanced expression of nesfatin/nucleobindin-2 in white adipose tissue of ventromedial hypothalamus-lesioned rats. Neurosci Lett 521: 46-51.

3. Shimizu H, Oh-I S, Hashimoto K, Nakata M, Yamamoto S, et al. (2009) Peripheral administration of nesfatin-1 reduces food intake in mice: The leptin-independent mechanism. Endocrinology 150: 662-671.

4. Ramanjaneya M, Chen J, Brown JE, Tripathi G, Hallschmid M, et al. (2010) Identification of nesfatin-1 in human and murine adipose tissue: a novel depot-specific adipokine with increased levels in obesity. Endocrinology 151: 3169-3180.

5. Yosten GL, Samson WK (2009) Nesfatin-1 exerts cardiovascular actions in brain: possible interaction with the central melanocortin system. Am J Physiol Regul Integr Comp Physiol 297: R330-336.

6. Osaki A, Shimizu H (2014) Peripheral administration of nesfatin-1 increases blood pressure in mice. Hypertens Res 37: 185-186.

7. Masuo K (2014) Nesfatin-1 could be a strong candidate obesity or diabetes medication, if blood pressure elevation can be controlled. Hypertens Res 37: 98-99.

8. Iwamoto T, Kita S, Zhang J, Blaustein MP, Arai Y, et al. (2004) Saltsensitive hypertension is triggered by $\mathrm{Ca} 2+$ entry via $\mathrm{Na}+/ \mathrm{Ca} 2+$ exchanger type-1 in vascular smooth muscle. Nat Med 10: 1193-1199.

9. Zhao Y, Ma X, Wang Q, Zhou Y, Zhang Y, et al. (2014) Nesfatin-1 correlates with hypertension in overweight or obese Han Chinese population. Clin Exp Hypertens.

10. Garty H, Palmer LG (1997) Epithelial sodium channels: function, structure, and regulation. Physiol Rev 77: 359-396.

11. Rossier BC (2014) Epithelial sodium channel (ENaC) and the control of blood pressure. Curr Opin Pharmacol 15: 33-46.

12. Fujita T (2010) Mineralocorticoid receptors, salt-sensitive hypertension, and metabolic syndrome. Hypertension 55: 813-818. 